\title{
De la eSPeCUlación a LA \\ CULTURA DE LA REHABILITACIÓN. PAX-Patios de LA Axerquía como estrategia ANTIGENTRIFICACIÓN EN ENTORNOS PATRIMONIALES
}

From speculation to the culture of rehabilitation.

PAX-Patios of Axerquía as an antigentrification strategy in heritage contexts

\section{Gaia Redaelli}

Arquitecta $\mathrm{PhD}$

Politecnico di Milano, Italia - Universidad de Sevilla, España gaiared@hotmail.com — http://orcid.org/0000-0002-0666-3660

RESUMEN: Frente a las dinámicas de fuerte tensión especulativa durante la burbuja inmobiliaria, dirigida fundamentalmente a la ciudad en expansión, es decir, a la ocupación de territorio y espacio público, hoy el ajedrez de los fondos de inversión parece dirigirse cada vez más al interior de la ciudad. Este artículo pretende abrir algunas pautas acerca de la relación actual entre Gentrificación y Derecho a la Ciudad, como deseo de una colectividad hacia el futuro de nuestro entorno urbano. En concreto, se analizarán algunas experiencias acerca de la rehabilitación urbana e innovación social en ciudades mediterráneas y, más en profundidad, la relación entre economía social y rehabilitación urbana de áreas de alto valor patrimonial. Con mayor detalle, se analizará la experiencia de reactivación urbana a través de procesos cooperativos en curso en el caso de PAX-Patios de la Axerquía, una estrategia de rehabilitación y reuso de casas de vecinos en el casco histórico de Córdoba como medida de cohesión social en una zona de alto valor patrimonial - reconocida por la Unesco en su componente material e inmaterial—, en riesgo de gentrificación.

Palabras clave: Rehabilitación urbana; innovación social; cooperativismo; gentrificación; derecho a la ciudad. 
ABSTRACT: Faced with the dynamics of strong speculative tension during the real estate bubble, aimed primarily at the expanding city, that is, the occupation of territory and public space, today the chess of investment funds seems to be increasingly directed towards the interior of the city. This article aims to open some guidelines about the current relationship between Gentrification and the Right to the City, as a desire for a collective towards the future of our urban environment. Specifically, some experiences about urban rehabilitation and social innovation in Mediterranean cities and, more in depth, the relationship between social economy and urban rehabilitation of areas of high heritage value will be analyzed. In more detail, the experience of urban revival will be analyzed through ongoing cooperative processes in the case of PAX-Patios de la Axerquía, a strategy of rehabilitation and reuse of neighboring houses in the historic center of Córdoba as a measure of social cohesion in an area of high heritage value -recognized by Unesco in its material and immaterial component-, at risk of gentrification.

KEYWORDS: Urban rehabilitation, Social innovation, Cooperativism, Gentrification, Right to the City.

\section{Gentrificación y Derecho a la ciudad}

$\mathrm{E}$ 1 concepto de gentrification nace en Inglaterra en los años 60 de mano de la experta en sociología urbana Ruth Glass. Estudia el fenómeno de expulsión forzosa de un sector social de un área urbana concreta y su sustitución por un colectivo de mayor capacidad adquisitiva. A menudo relacionada con el incremento de los flujos turísticos, la gentrificación es consecuencia también de las dinámicas del mercado residencial, de fondos interesados en nuevos contenidos y de proyectos de regeneración urbana de la administración. Así, «la substitución forzosa de la población en las áreas urbanas -ya sea a través de políticas públicas, ya sea a través de los mecanismos del mercado del suelo y la vivienda, ya sea a través de la combinación de ambos - resulta a menudo en un incremento de las desigualdades sociales y de la segregación urbana» (Indovina, Nel·lo; 2017). 
Si bien el concepto de gentrificación, cada vez más presente en ámbitos no sólo académicos, necesita de una lectura atenta y promenorizada — para evitar análisis y políticas equivocadas_-, es indudable que la actual dicotomía entre economía global y cultura local produce contradicciones y fenómenos especulativos allá donde la crisis ha sido más incisiva y, a la par, hay mayor patrimonio y potencial turístico, como sucede en las ciudades del Mediterráneo. Si durante la burbuja inmobiliaria el ajedrez de la especulación se jugaba sobre el modelo en expansión, ocupando territorio y espacio público, hoy la «nueva cuestión urbana» — y en especial «le disuguaglianze sociali e il loro dar luogo a forme evidenti di ingiustizia spaziale» (Secchi, 2013, p. 6) - se juega sobre todo en las zonas de mayor interés patrimonial, como los cascos históricos, es decir, en las propias casas de los habitantes.

La gentrificación — en su formato, hoy, más evidente de turistificaciónno sólo significa una sustitución de clase social en barrios concretos, sino también un cambio de uso prioritariamente residencial — que garantiza ciudades mixtas y complejas, tanto en términos residenciales como comerciales y de espacio público - al turístico, que prevé un uso de la ciudad de forma temporal. La consecuencia es doble. Por un lado, puede conllevar a la pérdida del «valor material del patrimonio», allá donde el formato de vivienda de uso turístico modifica de forma sustancial los edificios según parámetros generales y uniformados, por lo que la vivienda que buscamos en Lisboa a través de cualquier portal de la llamada «sharing economy» es muy parecida a la que podemos encontrar en París. Por otro lado, el monocultivo turístico incide en el «valor inmaterial del patrimonio», es decir, social y colectivo de nuestro entorno: el cambio de uso de las plantas bajas, por ejemplo, hacia comercios dirigidos a esos usuarios, termina expulsando al pequeño comercio que sirve a los residentes que, a su vez, son expulsados de sus casas.

No se trata sólo de considerar la capacidad de espacio público y equipamientos de soportar crecientes flujos turísticos, sino de entender que el mercado inmobiliario de zonas centrales y de interés - de forma aparentemente invisible en un primer momento - comporta una real pérdida de capacidad de acceso — en propiedad o en alquiler — por buena parte de la población en un círculo irreversible. La variación incontrolada del mercado inmobiliario según lógicas especulativas de fondos de inversión globales - 
de forma transitoria ligadas a la industria turística - produce cambios irreversibles en la relación entre ciudad y su ciudadanía: la «generalización de la gentrificación» debe entenderse como una «estrategia urbana global» (Smith, 2002).

Así, tras las reivindicaciones en defensa del «derecho a la vivienda» que han caracterizado en los últimos años el debate ciudadano, la gentrificación y el riesgo de pérdida de identidad y la homologación urbana y social se configuran entre los principales escollos para garantizar el «derecho a la ciudad», o un «deseo», puesto que como dice Harvey (2012):

El Derecho a la Ciudad es mucho más que un derecho de acceso individual o colectivo a los recursos que ésta almacena o protege; es un derecho a cambiar y reinventar las ciudades de acuerdo a nuestros deseos. Es, además, un derecho más colectivo que individual, ya que la reinvención de la ciudad depende inevitablemente del ejercicio de un poder colectivo sobre el proceso urbanizador. (Harvey, 2012: 20)

En una Europa en crisis, con una industria del turismo y fondos especulativos interesados en nuevos contenidos, las ciudades del Mediterráneo tienen que dar respuesta a la presión que la economía global ejerce en lugares de alto interés histórico y cultural. Se hace imprescindible la apertura de un debate hacia renovados paradigmas sobre el patrimonio urbano, su potencialidad económica y su papel social. Es necesario establecer mecanismos de gobernanza entre ciudad y ciudadanía que garanticen la rehabilitación y reactivación de un patrimonio arquitectónico que alumbra un patrimonio inmaterial: la convivencia de sus habitantes.

\section{Europa y patrimonio}

El difícil equilibrio entre economía global y dimensión local se evidencia, hoy, en varias ciudades, desde Lisboa a Berlín, desde Amsterdam a Nápoles, Atenas, París entre otras. En especial, en el ámbito mediterráneo, el valor patrimonial y la presencia de la industria turística como motor de una economía ajena a las lógicas locales generan incongruencias de forma más evidente en algunos casos. Venecia y Barcelona, dentro de sus amplias dife- 
rencias, resultan de interés por el grado de avance en cuanto a incidencia del turismo en su tejido urbano y social que la primera vive y ante el que la urbe catalana experimenta un intento de reacción desde lo público.

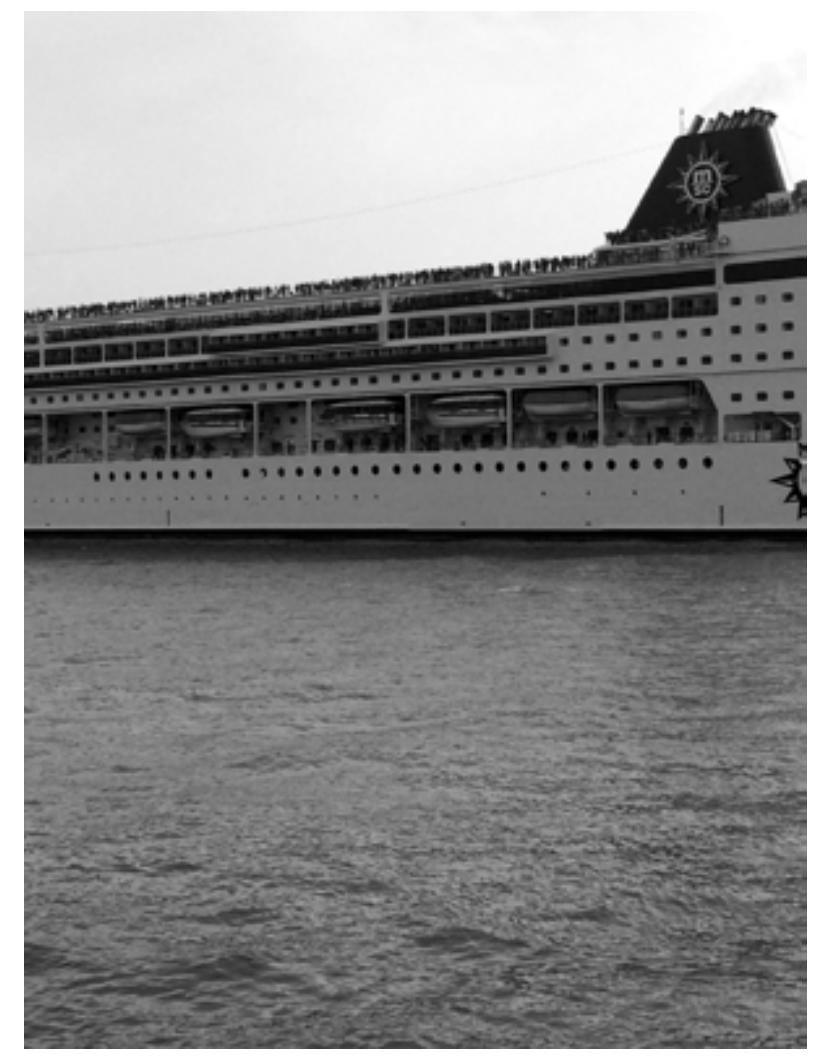

\section{Imagen 1. Turistas observan Venecia desde un crucero en la Laguna. Foto:} Gaia Redaelli.

La transformación de Venecia en un parque temático, principalmente debido al monocultivo turístico, es hoy el ejemplo excelente del riesgo que corren los cascos históricos europeos de alto valor patrimonial por la turistificación. La ciudad, con su delicada estructura y sus calles estrechas, evidencia una desmesurada afluencia de turismo de masas —en formato de ingresos diarios desde la península y por la llegada de cruceros en la Laguna - y una pérdida constante de residentes, un monocultivo que incide fuertemente en el mercado inmobiliario de una ciudad sin capacidad de expansión, además de en el uso de sus espacios públicos y comercios. 
Así, Venecia ha vivido un doble proceso de gentrificación: el primero, en los años setenta y ochenta, ha llevado a la sustitución de la población de personas mayores por una población de estudiantes, ligada al carácter universitario de la ciudad. En las últimas dos décadas, en cambio, Venecia va perdiendo población residente - también la estudiantil que, en todo caso, hace un uso «cotidiano» de la ciudad y sus servicios-, hacia una población flotante de carácter turístico cuyas necesidades se alejan mucho de la vida cotidiana. Se puede decir que, en Venecia, los apartamentos podrían dejar de tener cocina, ya que ningún turista la necesita para las dos noches que permanecerá en las islas venecianas, mientras, por el contrario, el comercio y el uso del espacio público van modulándose para dar respuestas a esos colectivos. Así, tal como se transforma físicamente, en su valor patrimonial material, también se va perdiendo la forma de convivencia, es decir el patrimonio inmaterial. La condición especial de la ciudad lagunary su significado emblemático a nivel europeo-, bien se resume en las reflexiones de Settis, que enlaza su condición histórica y su realidad actual de lo local a lo global, hacia esa cultura europea que busca una identidad:

Il futuro della città storica è un grande tema che si gioca non solo a Venezia e non solo in Italia, ma di cui Venezia può essere assunta a simbolo supremo. A ogni giorno che passa è sempre più urgente chiedersi come ciascuna città possa fondere il proprio capitale simbolico con il capitale civico dei cittadini, traducendolo in un consapevole diritto alla città e mettendolo a frutto. Come possa identificare forma fisica della città e la sua ragione etica, come possa fare del diritto alla città, alla funzione sociale della proprietà, al lavoro dei cittadini, la propria ragion d'essere e il proprio progetto. Se mai questo accadesse a Venezia, potrà accadere dappertutto. (Settis, 2015)

Consciente de que, «se Venezia muore», la siguiente podría ser ella, Barcelona, más que otras ciudades europeas, ha puesto el foco sobre este debate, desde que las anteriores elecciones municipales dieron paso a un gobierno que pone en primer plano el Derecho a la Vivienda, pero también el Derecho a la Ciudad. Al convertir el turismo en un motor económico principal, Barcelona no sólo ha ido perdiendo la primacía como ciudad de la cultura urbana que adquirió a principios de los noventa, sino que parece haberse hecho esclava de su propia imagen, mecanismos de gentrificación incluidos. El 
Ayuntamiento trata de paliar esos efectos y disminuir la desigualdad social a partir del reconocimiento de su plasmación sobre el territorio con el Pla de Barris - que interviene en zonas de la ciudad prioritarias por su vulnerabilidad - pero, a la vez, se propone impulsar medidas y políticas públicas que eviten la expulsión de la población de ámbitos más céntricos por un turismo descontrolado y por fondos buitre. Barcelona ha abierto, en 2017, un Espacio de Corresponsabilitat sobre Gentrificació, una mesa de coordinación transversal, con presencia de diferentes áreas municipales - Urbanismo, Vivienda, Bienestar Social, Turismo, Hacienda, Comercio, entre otras- que estudia las dinámicas de la urbe para generar acciones que palien la gentrificación. De forma complementaria, y en aras a abrir una red de colaboración internacional en estos temas, se ha redactado un estudio sobre políticas y medidas anti-gentrificación en ciudades europeas, relevando las Buenas Prácticas impulsadas por administraciones públicas, universidades y movimientos sociales conformadas en un «Atlas de la A-Gentrificación». ${ }^{1}$

Ejemplo de desarrollo urbano y de espacio público en su etapa olímpica — con el denominado «modelo Barcelona»—, la ciudad, aún con diferencias sustanciales con Venecia, padece hoy un importante revés, ya que:

En este momento los procesos de sustitución de la población son suscitados no tanto a través de actuaciones para la residencia permanente en el mercado residencial, sino sobre todo a través del impacto de la actividad turística. La evolución, ya madura, del mercado inmobiliario de Venecia y la rápida transformación de algunos barrios de Barcelona en los últimos años (Barceloneta, el Born) son excelentes ejemplos de la capacidad disruptiva del turismo sobre el mercado residencial (Indovina, Nel·lo; 2017).

Venecia y Barcelona significan un caso comprometido y un intento desde lo público de prevención para que el fenómeno no se extienda. Sin embargo,

1. Se reenvía al trabajo por la autora realizado entre julio 2017 y enero de 2018 de análisis de Best Practices en ciudades europeas que han emprendido políticas y acciones anti gentrificación de cara a su posible aplicación en Barcelona. Se han analizado Lisboa,Venezia, Milano, Berlín, Madrid, Valencia, Marsella, Cádiz, Atenas, París, Viena, Nápoles y Londres a través de entrevistas a personas de la administración municipal, del mundo académico y de los movimientos sociales. Publicado en G. Redaelli. (2018). 
resulta especialmente interesante analizar si es posible contener esos procesos en acciones compartidas entre ciudad y ciudadanía, ya que la crisis ha afectado a ambos. Es decir, si es posible, desde el valor patrimonial observado bajo enfoques nuevos y en su componente social, el fomento de acciones de rehabilitación urbana e innovación social para usos contemporáneos en el respeto de su valor patrimonial.

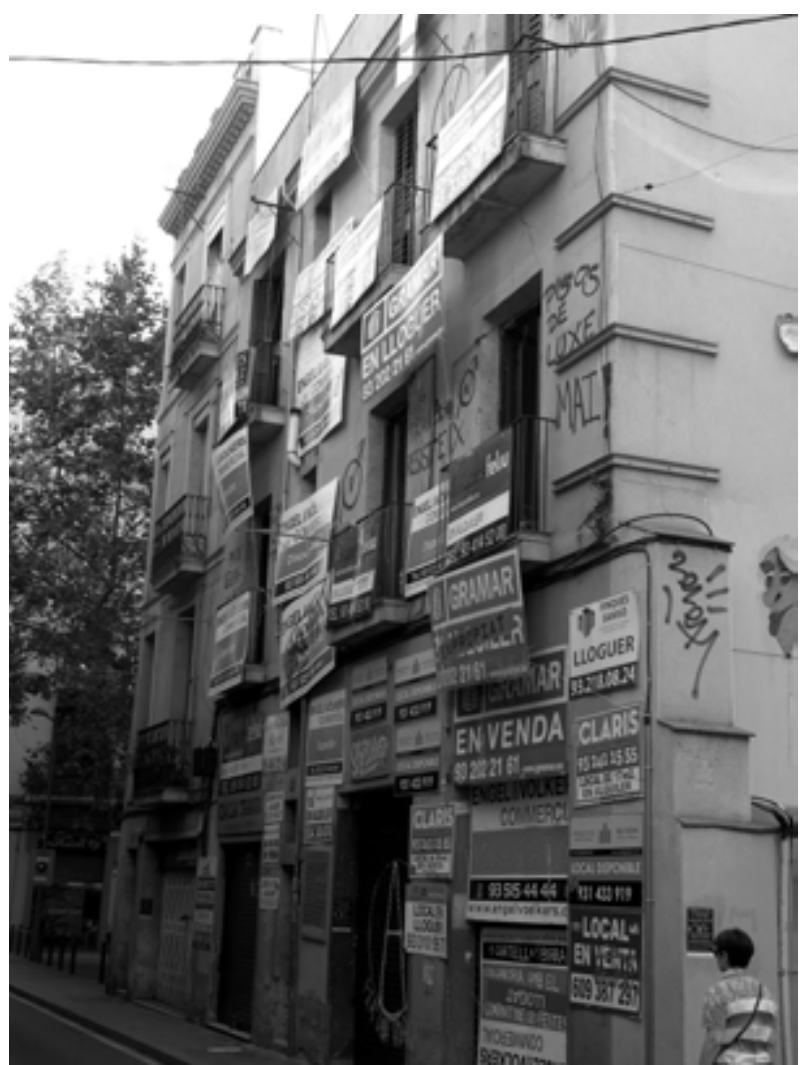

Imagen 2. Pisos en venta en Barcelona. Foto: Vito Redaelli.

\section{Rehabilitación urbana e innovación social}

En 2005, el Consejo de Europa aprobó en la ciudad portuguesa de Faro el «Convenio marco sobre el valor del patrimonio cultural para la sociedad» que significa un importante cambio de la noción de patrimonio. Entre las definiciones incluidas en su artículo dos, la Convención renueva la de patrimonio cultural e introduce la de comunidad patrimonial: «por patrimonio cultural se entiende un conjunto de recursos heredados del pasado que las personas identifican, con independencia de a quién pertenezcan, como reflejo 
y expresión de valores, creencias, conocimientos y tradiciones propios y en constante evolución» y «una comunidad patrimonial está compuesta por personas que valoran aspectos específicos de un patrimonio cultural que desean conservar y transmitir a futuras generaciones, en el marco de la actuación de los poderes públicos». ${ }^{2}$

Si la precedente «Convención para la salvaguardia del patrimonio arquitectónico en Europa», suscrita en Granada en 1985, incidía en la necesidad de inventariar, conservar y difundir la arquitectura histórica como «expresión insustituible de la riqueza y diversidad del patrimonio cultural de Europa, un testimonio inestimable de nuestro pasado y un bien común para todos los europeos», Faro señala nuevos retos. Nace de la observación de algunas acciones promovidas por la ciudadanía — en un formato bottom-up - y se propone poner en sintonía instituciones y sociedad civil alrededor de una renovada noción de patrimonio: de elemento puramente conservativo del pasado, y, por tanto, de carácter contemplativo o mercantil/turístico, se transforma en algo vivo, memoria, pero, al mismo tiempo, futuro de una colectividad que es la verdadera activadora de un habitat sostenible. En definitiva, la Convención de Faro reconoce la dimensión social del patrimonio, un recurso estratégico para Europa hacia un crecimiento inteligente, sostenible e integrador.

A partir del reconocimiento del «patrimonio inmaterial» (Paris, 2003) y de cara a entender la estricta relación entre «patrimonio»y «ciudad», o según lo que nos ocupa, «derecho a la ciudad», es relevante recordar que la propia noción de patrimonio aprobada en 2011 por la UNESCO trasciende el edificio en su singularidad —así como su valor mercantil— y alcanza la dimensión urbana, social y económica:

La noción de «paisaje urbano histórico» responde al objetivo de preservar la calidad del medio en el que viven las personas, mejorando la utili-

2. Convenio marco del Consejo de Europa sobre el valor del patrimonio cultural para la sociedad, Convención de Faro (STCE $n^{\circ} 199$ ) Faro, 27/10/2005, en vigor desde 01/06/2011, firmado por España el 12/12/2018. Para el concepto de Patrimonio Inmaterial se remite a Unesco, Convención para la salvaguardia del Patrimonio cultural Inmaterial, 2003 y Recomendación sobre el paisaje urbano histórico, con inclusión de un glosario de definiciones, 2011. 
zación productiva y sostenible de los espacios urbanos, sin perder de vista su carácter dinámico, y promoviendo la diversidad social y funcional. En ella confluyen los objetivos de la conservación del patrimonio urbano y los del desarrollo social y económico. Es un planteamiento basado en una relación equilibrada y sostenible entre el medio urbano y el medio natural, entre las necesidades de las generaciones presentes y venideras y la herencia del pasado.

En la búsqueda de la compatibilidad entre lo global y lo local, algunos ejemplos en ciudades mediterráneas intentan romper los esquemas preestablecidos para abrir nuevos mecanismos que, aún experimentales, eviten el dudoso solapamiento entre turismo y cultura. Analizamos dos casos de estudio que - en condiciones geográficas diferentes - buscan efectos comparables en cuanto a rehabilitación urbana e innovación social. El primero, en la ciudad de Marsella, promueve acciones relacionadas con la economía social y el turismo local en un entorno periférico. El segundo actúa de forma bottom-up para evitar la pérdida de identidad de un casco histórico de alto valor patrimonial en Córdoba. El análisis de estos casos $-\mathrm{y}$ en especial el caso de Córdoba, en cuanto experiencia en curso y directa por parte de un grupo de investigadores, entre ellos, quien escribe como investigación aplicada al territorio-, plantea cuestiones relevantes a la hora de intervenir en entornos urbanos sensibles a través de operaciones de regeneración urbana e innovación social. Ambas iniciativas, de hecho, son parte de la Faro Convention Network del Consejo de Europa, en cuanto implementación en el territorio y formato bottom-up de los principios de la Convención de Faro.

\subsection{Marsella: barrios vulnerables y patrimonio}

Marsella fue, en 2013, Capital Europea de la Cultura, ocasión que comportó relevantes cambios de renovación urbana del casco histórico y nuevos contenedores culturales. La operación privilegió la renovación de la zona del puerto antiguo con intervenciones en el espacio público y con la rehabilitación de edificios históricos significativos para su trasformación en centros culturales, así como la rehabilitación de los almacenes del puerto destinados a centro comercial. Estas operaciones urbanas han sido, posiblemente, la pla- 
taforma para fenómenos de gentrificación incipiente. Un fuerte desequilibrio entre centro y periferia, y un desarrollo que toma como ejemplo a la Barcelona olímpica — con la que comparte, sin duda, algunas característicasestán transformando la ciudad con una lenta, pero inexorable, expulsión del vecindario de las zonas centrales. El aumento del turismo cultural y, prioritariamente, urbano está promoviendo la rehabilitación de edificios alrededor del puerto antiguo para su incorporación al sistema de alojamiento turístico.

En 1995 se inició en Marsella un camino experimental para la valorización del patrimonio integrado, a través de una colaboración entre el Consejo de Europa, la administración municipal y la universidad. Esta mesa - coordinada por el Ayuntamiento - funcionó con intensidad durante quince años, convirtiéndose en una figura de referencia en el servicio público que se relaciona con el patrimonio cultural. Tras el intenso trabajo realizado y gracias a la solicitud de la comunidad local, la mesa promovió y consiguió algunas pautas clave: la adhesión del alcalde de Marsella a los principios del Convenio de Faro; la constitución de una comisión que reúne a políticos, instituciones y ciudadanos; la continuidad a estas acciones a través del compromiso de una colaboración entre investigadores y residentes.

En enero de 2011, se constituyó la cooperativa de habitantes Hotel du Norte con el objetivo de aprovechar el patrimonio del arrondissement de Marsella Vitrolles para mejorar la vida de los que viven y trabajan en los barrios vulnerables del norte de la ciudad. Desde entonces la cooperativa de habitantes promueve la hospitalidad y el redescubrimiento del patrimonio en los distritos al Norte de Marsella, considerados peligrosos e impracticables para turistas y ciudadanía. La cooperativa, conformada por 70 personas y entidades, ofrece alojamiento a turistas en viviendas o habitaciones (i), paseos urbanos patrimoniales alternativos fuera del casco histórico (ii), visita a artesanos locales (iii) y venta de productos locales y libros (iv). Ha elegido como horizonte el euro-mediterráneo, cultivando y promoviendo una red de contactos con otros movimientos ciudadanos que comparten sus objetivos. El principio fundamental reside en la traducción de la industria turística, basada sobre lógicas globales, en una economía local que sepa generar beneficios a sectores sociales y urbanos de bajos ingresos y ponga las bases para un turismo más sostenible. 
Generar un cambio de paradigma sobre qué es patrimonio y sobre su componente social se ha ido fortaleciendo en el caso de Hotel du Nord — no sin dificultades derivadas de la competencia de una falsa sharing economy como la de Airb\&b. Tras casi una década de andadura, la experiencia de Marsella se abre a una visión más amplia. La cooperativa sigue desarrollando y construyendo nuevas alianzas, potenciando su impacto social y económico en el territorio y, ahora, en colaboración con el Estado francés, se ha transformado en una start up de cooperativas similares en el territorio nacional bajo el nombre Oisseaux de passage. La plataforma generada busca así poner en red y dar visibilidad a acciones ciudadanas y emprendimientos turísticos más sostenibles e integradores del patrimonio cultural y de la ciudadanía a partir de las experiencias de comunidades patrimoniales según lo indicado por Faro. ${ }^{3}$

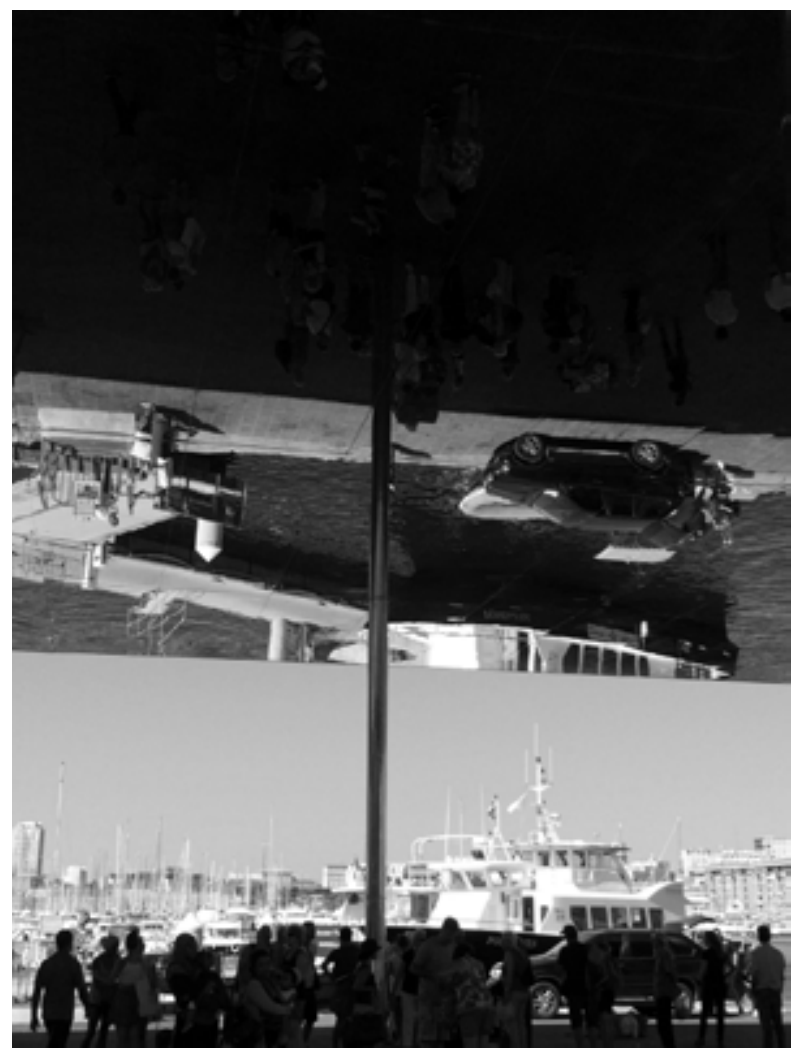

Imagen 3. Turistas debajo de la marquesina de Norman Foster, símbolo de las intervenciones en el Puerto Viejo de Marsella. Foto: Gaia Redaelli.

3. Para más información sobre estas iniciativas, se invita a la consulta de las páginas web http:/hoteldunord.coop/ y https://lesoiseauxdepassage.coop/ 


\subsection{Córdoba: cascos históricos y patrimonio}

La dimensión urbana patrimonial se hace especialmente presente en ciudades cuyo casco histórico está declarado Patrimonio de la Humanidad por la UnESCO. Córdoba ha obtenido recientemente el cuarto reconocimiento de la UNESCO: tras la declaración de Patrimonio de la Humanidad en 1984 de la Mezquita, se añade en 1994 la declaración de una gran parte del centro histórico. Posteriormente, en 2012, la UnESCO reconoce la Fiesta de los Patios de Córdoba como patrimonio inmaterial y, finalmente, en 2018, la ciudad califal de Madinat al-Zahra viene inscrita en la Lista del Patrimonio Mundial. Con el creciente turismo, ligado a lógicas globales, y con una escasa actividad industrial, Córdoba se dirige hacia caminos de monocultivo turístico que inciden en su tejido urbano, gentrificándose de forma gradual en un proceso que, como en otros casos, puede acelerarse repentinamente si las condiciones de regulación pública y fondos buitre lo facilitan. Para evitar lo que ha sucedido en otras ciudades, como Venecia, Barcelona o Lisboa, por mencionar algunas, Córdoba se encuentra en una encrucijada: considerar su valor patrimonial en clave mercantil y, por lo tanto, explotarlo para producir riqueza inmediata, pero dejando consecuencias importantes en el mediano y largo plazo, o introducir los mecanismos de cogestión entre ciudad y ciudadanía sugeridos por Faro para hacer que los cambios necesarios en la contemporaneidad sean compatibles con el valor social del «patrimonio como bien común». ${ }^{4}$

Para repensar su historia en clave actual - abriéndose a ese cambio de paradigma que, además de responder a dinámicas de economía global desde lo local, atribuya al patrimonio un papel de activador de la ciudad por y para la ciudadanía-, es imprescindible buscar fórmulas novedosas que permitan prevenir fenómenos especulativos en el interior de la ciudad actualizando el valor colectivo del patrimonio y su potencial económico, siempre y cuando se reconozca su dimensión social. Desde 2018, PAX — acrónimo de Patios de

4. Veáse contenido y mesas redondas del Simposio internacional «Patrimonio como Bien común. La Cultura del espacio público» celebrado en Córdoba los días 12, 13 y 14 de diciembre de 2018. Organizado por Delegación de Cultura de la Diputación de Córdoba. Dirección: Gaia Redaelli. En: https://patrimoniocomobiencomun.home. blog/. También se remite, acerca de las consecuencia de un exceso de explotación turística de los patios, al Foro de IESA-CSIC y la Cadena SER (2018): ¿Qué hacemos con los patios?, Córdoba, marzo de 2018 y al I Congreso internacional Patios de Córdoba, Universidad de Córdoba, 14-16 de noviembre de 2018. 
la Axerquía - es una estrategia de reactivación urbana a través de procesos cooperativos, que persigue que la mejora sostenible del centro histórico no suponga la alienación social de zonas patrimoniales por el incremento de la presión turística. ${ }^{5}$ La rehabilitación del barrio de la Axerquía, amenazado por la gentrificación, busca la recuperación del valor medioambiental y social de la urbe mediterránea y la actualización de su historia en clave contemporánea a través de la innovación social con la ciudadanía. La presencia de tipologías tradicionales - como las casas-patio o las casas de vecinos-, en muchos casos vacías o infrautilizadas como resultado de la burbuja, sugiere mecanismos de gobernanza que garanticen la rehabilitación y reactivación de este patrimonio arquitectónico y su valor inmaterial. El fomento de acciones que propicien la adquisición de casas-patio en desuso para la sucesiva rehabilitación por cooperativas habitacionales en cesión de su uso permite la salvaguarda de un patrimonio universal y su valor ambiental, promover empleo local y facilitar un tejido social, asociativo y solidario en el centro de la ciudad para un uso colectivo de los patios, tal y como fue reconocido por la UNESCO.

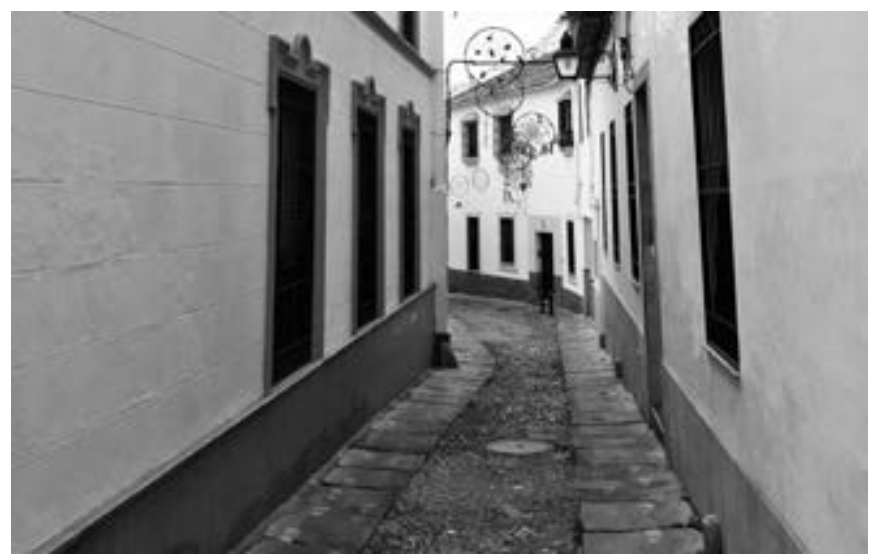

Imagen 4. Calle del barrio de la Axerquía en Córdoba. Foto: Carlos Anaya.

5. La estrategia en cuanto implementación de gobernanza innovadora se conforma en 2018 como «Asociación PAX - Patios de la Axerquía», co-fundada por Gaia Redaelli (Presidenta), Jacinta Ortiz (Secretaria) y Carlos Anaya (Tesorero). El proyecto fue inicialmente elaborado y luego presentado a Urban Innovative Action (2016) por los autores: Gaia Redaelli (Studio Redaelli Speranza architetti associati: Gaia Redaelli, Vito Redaelli, Anna Speranza), dirección. Jacinta Ortiz, coordinación. Carlos Anaya, Eva Morales, Felipe García, Manuel Rodríguez, equipo técnico. Partners: Ayuntamiento de Córdoba (Jefe de fila), Instituto Andaluz de Patrimonio Histórico, Universidad de Córdoba, Universidad de Sevilla, FAECTA. Para información sobre la Asociación y sus acciones, consultar www.patiosaxerquia.eu 


\section{PAX - Patios de la Axerquía, estrategia de reactivación urbana con procesos cooperativos}

Un grupo multidisciplinar de facilitadores, del ámbito de la arquitectura y la antropología, funda en abril de 2018 la Asociación PAX-Patios de la Axerquía como una herramienta para impulsar el proceso de innovación cultural y social e involucrar a asociaciones locales y ciudadanía, entidades públicas y privadas, mundo de la investigación en la práctica experimental acerca del casco histórico en un entorno urbano de alto valor y en riesgo de gentrificación. El re-uso de la ciudad existente, en su valor patrimonial material e inmaterial ligado a las casas de vecinos en un casco objeto de transformación por un creciente turismo, ha sido el impulso para generar una estrategia en cuanto a cultura urbana, a través de la actualización de los patios con procesos de innovación social y cooperativos para su uso residencial.

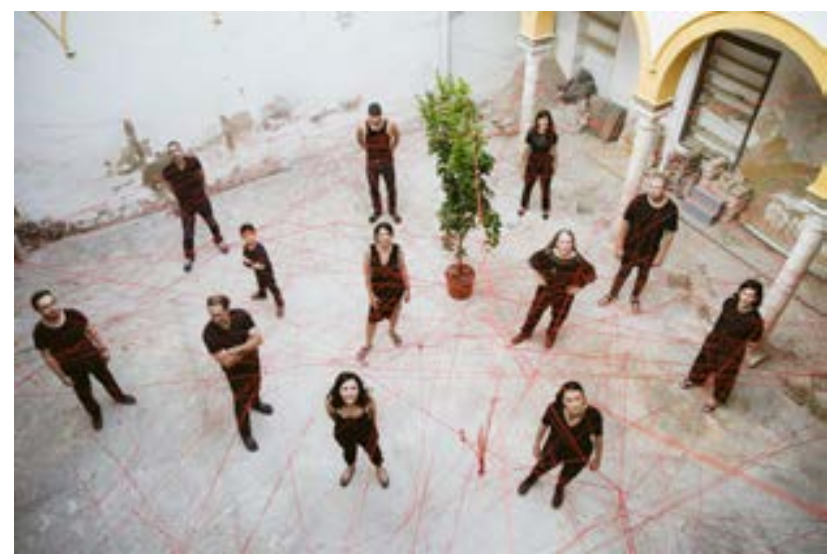

Imagen 5. Miembros del colectivo PAX durante una instalación para la Xv Biennal Internacional de Arquitectura de Venecia.

De acuerdo al doble carácter del patio de Córdoba, y a los principios de la Convención de Faro, la Asociación desarrolla dos ejes de acciones para reforzar la cultura de la rehabilitación en la ciudadanía:

- Patrimonio inmaterial. Acciones acerca de los patios:

- Estas actividades se centran en fomentar el empoderamiento de la comunidad en la reactivación de su patrimonio como valor inmaterial, potenciando el concepto de comunidad pa- 
trimonial, que reconozca los valores sociales y la convivencia alrededor del patio y en la ciudad mediterránea.

- Patrimonio material. Acciones sobre los patios:

- Estas acciones van dirigidas a promover la rehabilitación de las casas de vecinos en desuso a través de procesos cooperativos, que permitan contener su transformación en edificios turísticos y mantener su uso residencial, además de facilitar los mecanismos productivos relacionados con la rehabilitación.

La finalidad y unicidad de PAX - frente a otras experiencias de cooperativismo como alternativa a la propiedad y al alquiler- es que interviene en un entorno patrimonial de alto valor - actualizando la convivencia que caracteriza el patio y la ciudad mediterránea - y se propone ahora constituirse en una start-up, que une las cooperativas de vivienda, de rehabilitación y de servicios para generar una micro-economía local en un proceso piloto de la economía social como agente de rehabilitación urbana. Para eso, colabora en formatos diferentes con instituciones del ámbito de la administración, de la economía social, de la universidad y, sobre todo, con las personas y ciudadanía que se suman al proyecto que abarca distintas dimensiones. Por un lado, la dimensión ambiental (i), densificando la ciudad y poniendo en valor la red de elementos «verdes» de los patios — como green-cell a sistema de una verdadera ecología urbana mediterránea - que evita el efecto de isla de calor y disminuye el consumo energético; la dimensión económica (ii), a través de la inclusión de la economía social, incorporando a los colectivos ciudadanos como protagonistas en el diseño e implementación del proyecto, y promoviendo nuevas empresas sociales como base para una microeconomía y empleo local; la dimensión sociocultural (iii), en cuanto que la creación de cooperativas supone la apertura a nuevas formas de habitar, donde la recuperación de los espacios comunitarios es central y garantiza la pervivencia y autenticidad de los valores culturales del patrimonio; por último, la dimensión tecnológica (iv), en cuanto que el proceso promueve las herramientas para rehabilitar desde criterios de uso de materiales tradicionales y de eficiencia 
energética y reducción de la demanda en el respeto del patrimonio desde su componente constructiva.

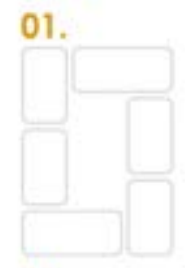

04.
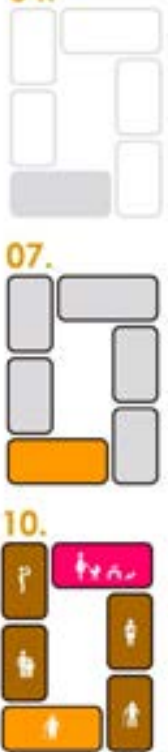
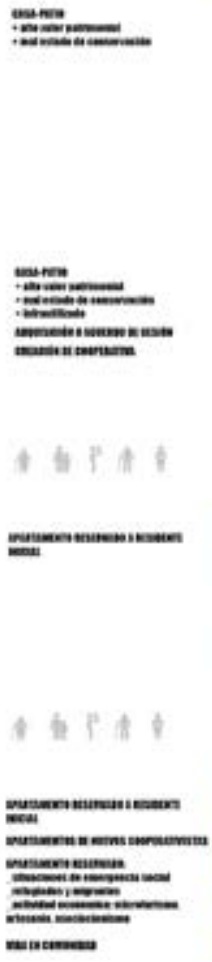

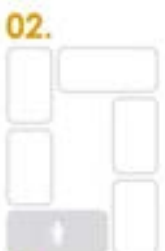

05.
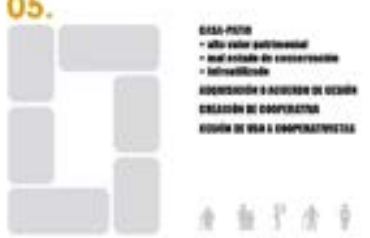

08.
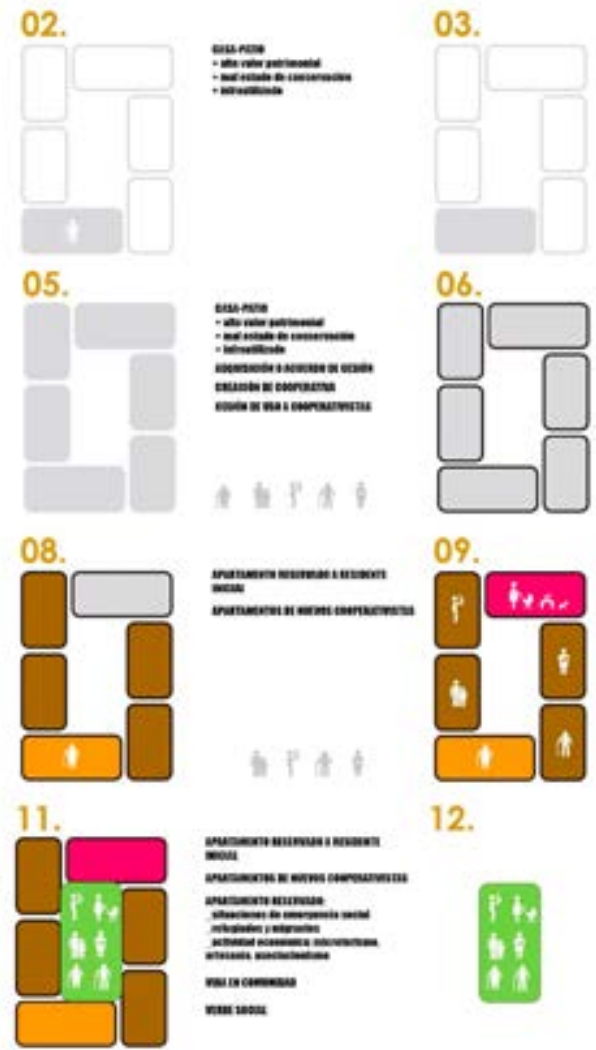

06

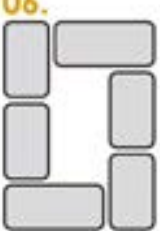

09

12.
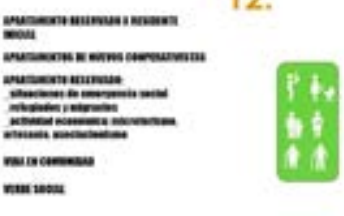
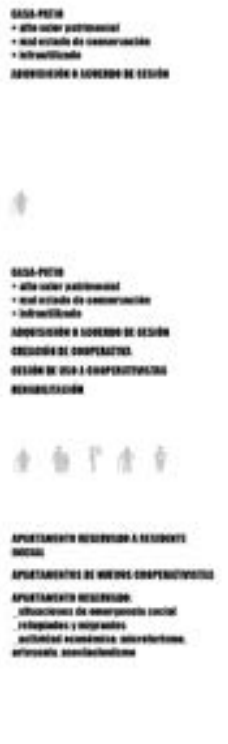

Imagen 6. Esquema de funcionamiento de cesión de uso de una casa patio. Fuente: PAX.

Los objetivos de la estrategia son múltiples y sinérgicos:

1. Promover un cambio de modelo hacia la cultura de la rehabilitación patrimonial de la ciudad existente que frene el consumo especulativo de territorio en el entorno urbano, apostando por la regeneración urbana y ambiental del centro histórico y su reactivación social.

2. Mantener el carácter residencial del casco, a través de la adquisición de edificios en desuso para su rehabilitación, contribuyendo a la densificación de la ciudad, a mantener la población local y a poner en valor un patrimonio - casas patio y casas de vecinos-, que repre- 
sentan la estructura urbana del barrio y son una forma de relacionarse entre vecinos.

3. Promover la creación de formas habitacionales de cooperativas de vecinos y vecinas a través de la cesión de uso de edificios para su rehabilitación y fomentar la incorporación de la economía social en la regeneración urbana del centro evitando su gentrificación.

4. Fomentar la generación de empleo local, y especialmente el empleo femenino, a través de la inclusión de empresas y cooperativas ligadas a la rehabilitación con especial atención a la población local como forma de generar empleo en el barrio.

5. Potenciar la red de espacio público como «patio colectivo» y reconocer el valor ambiental que significa en Córdoba la red de casas-patio, así como su papel en el control y reducción del consumo energético con medidas pasivas y del cambio climático.

6. Insertar a Córdoba en el debate europeo sobre la reactivación urbana sostenible de tejidos históricos, así como la inclusión de la economía social en el ámbito de la política de vivienda y de regeneración urbana para su transferencia a otras realidades mediterráneas.

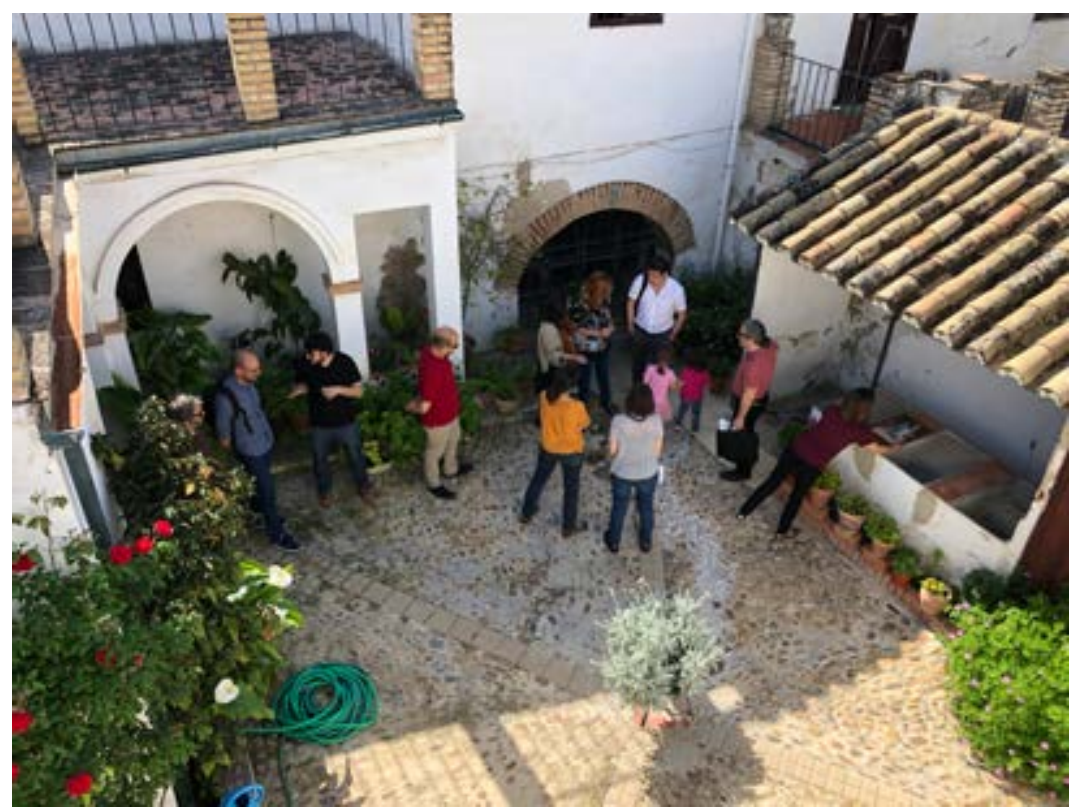

Imagen 7. Visita de miembros del Consejo de Europa a Pax, en el marco de la Faro Convention Network. 


\section{Primeros alcances de la estrategia PAX}

Como parte de la Faro Convention Network del Consejo de Europa, PAX busca una corresponsabilidad y cogestión que permita introducir dinámicas globales protegiendo y poniendo en valor las culturas locales al facilitar esa comunidad patrimonial, como «deseo» de ciudad a partir de sus valores patrimoniales de convivencia. Por lo tanto, se trata de una experiencia de abajo hacia arriba, trabajando en una relación con diferentes partes interesadas, como las Asociaciones de Vecinos y los grupos de ciudadanos interesados en crear una Cooperativa PAX, pero también participa como facilitador de proceso de cogestión con instituciones públicas y privadas para los aspectos multidisciplinarios y multinivel de la estrategia: en el campo del patrimonio material, el Instituto Andaluz del Patrimonio Histórico, de la Consejería de Cultura de la Junta de Andalucía y la Universidad de Sevilla; en el de lo inmaterial, la Universidad de Córdoba y el Instituto de Estudios Sociológicos Avanzados del Consejo Superior de Investigaciones Científicas; el Ayuntamiento de Córdoba; FAECTA. ${ }^{6}$

6. En aras a generar acciones multinivel, PAX colabora con varias entidades. El Instituto Andaluz del Patrimonio Histórico, reconoce PAX como buena práctica en «Redactivate» y colabora para actualizar el Atlas Digital del Patrimonio Inmaterial y preparar cursos para el uso de técnicas tradicionales, como la Cal en la rehabilitación; además, se han presentado conjuntamente varios proyectos de investigación. La Universidad de Córdoba, con el grupo de investigación Etnocórdoba, está trabajando en los patios desde un punto de vista antropológico y está colaborando con PAX en los aspectos sociales en relación con la comunidad local. En colaboración con PAX, investigadores de la Universidad de Sevilla, entre julio y agosto de 2017 y sucesivamente entre julio y septiembre de 2019, han monitorizado en la Axerquía las condiciones bioclimáticas en tres casas-patio, cuya presencia llega a reducir la temperatura entre 6 y 12 grados sin sistemas activos de refrigeración. El Instituto de Estudios Sociológicos Avanzados del Consejo Superior de Investigaciones Científicas está creando un mapa de tendencias demográficas y viviendas de uso turístico en Córdoba utilizando datos anuales sobre los residentes. Por último, el Ayuntamiento de Córdoba está redactando una estrategia de gestión del casco histórico, tal y como requiere la UNESCO, en la que PAX aportará experiencias desde la sociedad civil para preservar los valores patrimoniales frente a los especulativos. 


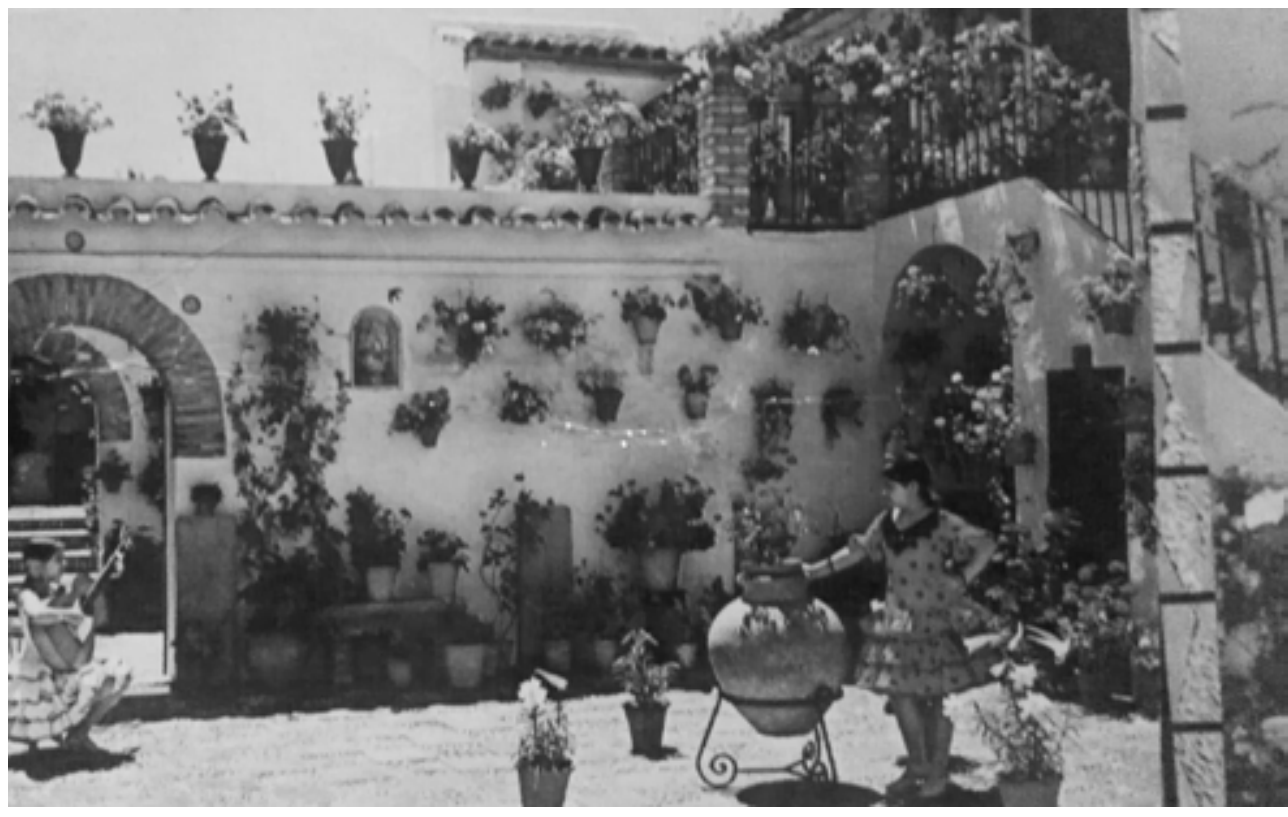

Imagen 8. Imagen histórica de la casa en calle Montero, primera cooperativa PAX.

Tras su constitución como Asociación, PAX ha realizado varias acciones de carácter cultural, tejiendo relaciones sobre todo con entidades locales y ciudadanía, también a través de la constitución, en 2018, del «Foro por el Derecho a la Ciudad de Córdoba» como lugar para compartir una idea común de futuro de la ciudad y la elaboración, entre otros, de un Acuerdo ciudadano. ${ }^{7}$

En junio de 2018 se ha creado la primera cooperativa que, bajo el nombre de PAX Astronautas, está conformada por seis hogares de personas de entre 35 y 45 años con descendientes de entre cuatro y siete años. El valor educativo que conlleva compartir el patio ha sido el principal motor, por lo que este grupo local se ha acercado al proyecto y, en enero de 2019, ha adquirido con financiación de Fiare Banca Ética, la casa de vecinos del siglo XVIII en la calle Montero, 12, en pleno barrio de la Axerquía. Este primer patio,

7. El «Foro del Derecho a la Ciudad de Córdoba», desde 2018, es una iniciativa transversal y multinivel de colaboración entre diferentes organizaciones, como la Asociación PAX, las asociaciones vecinales, la Universidad de Córdoba y otras asociaciones e iniciativas socioculturales, así como la ciudadanía de Córdoba. El objetivo principal es promover un consenso sobre el futuro de la ciudad de manera sostenible, plasmado en un primer documento participado en formato de «Acuerdo ciudadano». 
rescatado por la cooperativa PAX de un futuro cierto como hotel - gracias también a las dos propietarias que han preferido vender a la cooperativa antes que a un inversor externo por entender que las jóvenes parejas iban a recrear aquel modelo de convivencia que habían experimentado en su infancia-, será especialmente significativo, ya que se trata de un edificio catalogado de alto valor patrimonial, ganador en varias ocasiones del premio de la fiesta de los patios, y por la atención al valor ecológico del mismo, gracias a sistemas de rehabilitación tradicional, como la cal, junto a sistemas de calefacción/ refrigeración de energía verde, a través de aerotermia. Además, PAX va recopilando mapas del edificio, documentos, planos históricos, así como narrativas de las personas que han vivido y que son voces de una memoria viva de la casa, en la que han llegado a vivir 18 familias en situación de hacinamiento. Con esta primera obra, iniciada en febrero de 2020, PAX también fomenta el empleo local, a través de la contratación de la rehabilitación a una pequeña empresa de Córdoba y fomentando, por tanto, un tejido productivo ligado a la rehabilitación, en muchos casos, poco visible y desestructurado, como fuente de empleo sostenible en Córdoba.

Gracias a encuentros locales con la ciudadanía, otros grupos de personas van conformándose como posibles cooperativas, entre ellas, mujeres mayores en procesos de envejecimiento activo (bajo el nombre de PAX Patias) o entidades de carácter sociocultural que buscan aunar en el patio funciones habitacionales con actividades productivas, en especial de la economía social, entendiendo el papel de reactivación urbana más allá del puro residencial. Fundamental resulta la relación con las asociaciones vecinales del casco histórico con el fin de realizar, conjuntamente, un mapa de los bienes disponibles, así como aunar esfuerzos y cohesión social, sobre todo entre las personas mayores que viven en el centro y que, en muchos casos, se ven obligadas a dejar sus viviendas por no tener los recursos para su rehabilitación.

En 2019, la World Monument Fund —principal organización no-profit en New York que promueve a nivel mundial acciones de puesta en valor del patrimonio - incluyó, a instancias de la Asociación PAX -Patios de la Axerquía, a esta zona de Córdoba entre los 25 bienes patrimoniales en el mundo a preservar por su peligro de homologación y gentrificación. Tras dar el primer paso y generar la primera rehabilitación de una casa tan significativa, PAX 
afronta un nuevo reto: transformarse en los próximos años en una start-up de regeneración urbana e innovación social, como cooperativa de segundo nivel bottom up en entornos de alto valor patrimonial y que una a entidades públicas y privadas en un camino compartido para el futuro de una zona tan delicada entre lo global y lo local.

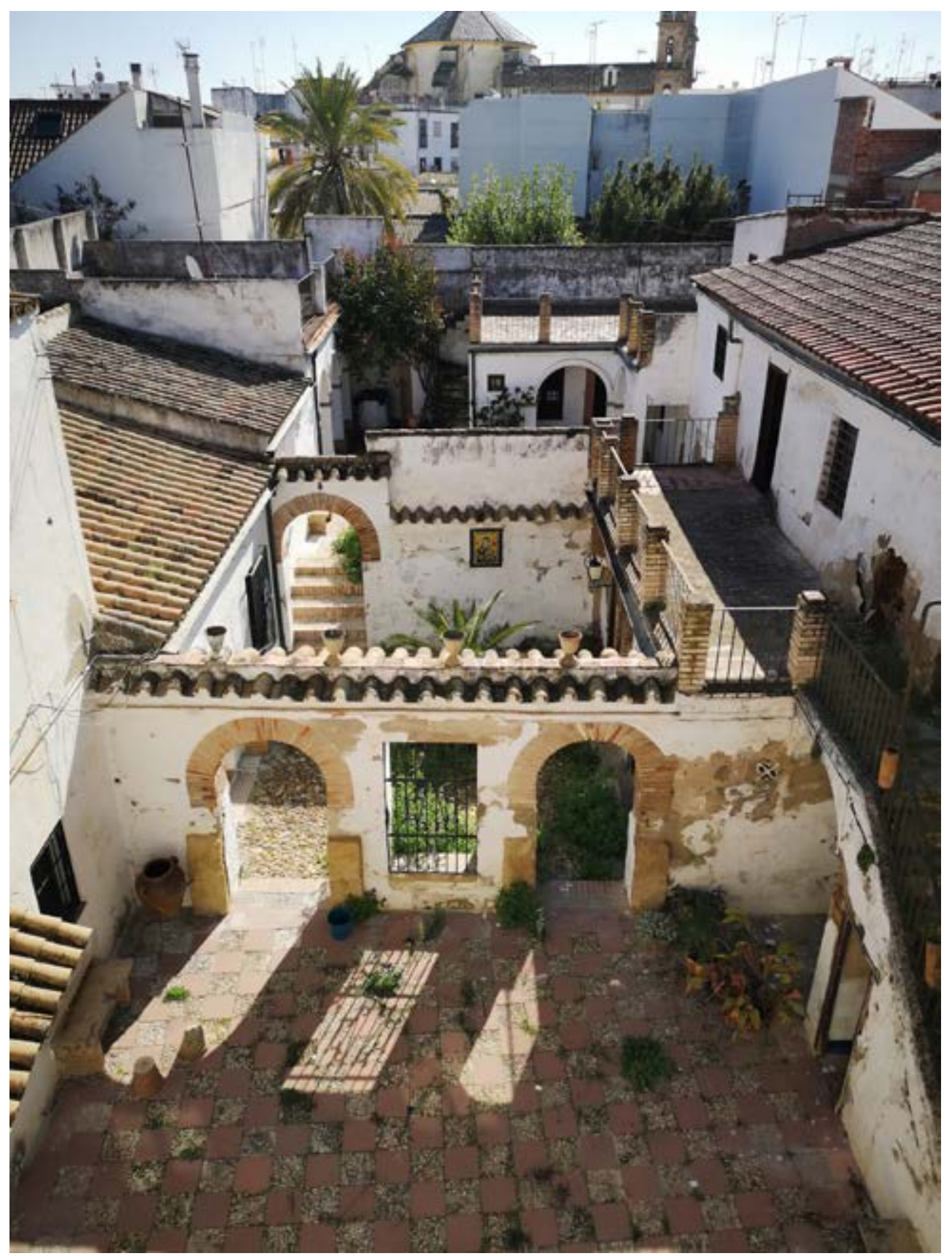

Imagen 9. Imagen actual de la casa en calle Montero, primera cooperativa PAx, antes de la rehabilitación. 

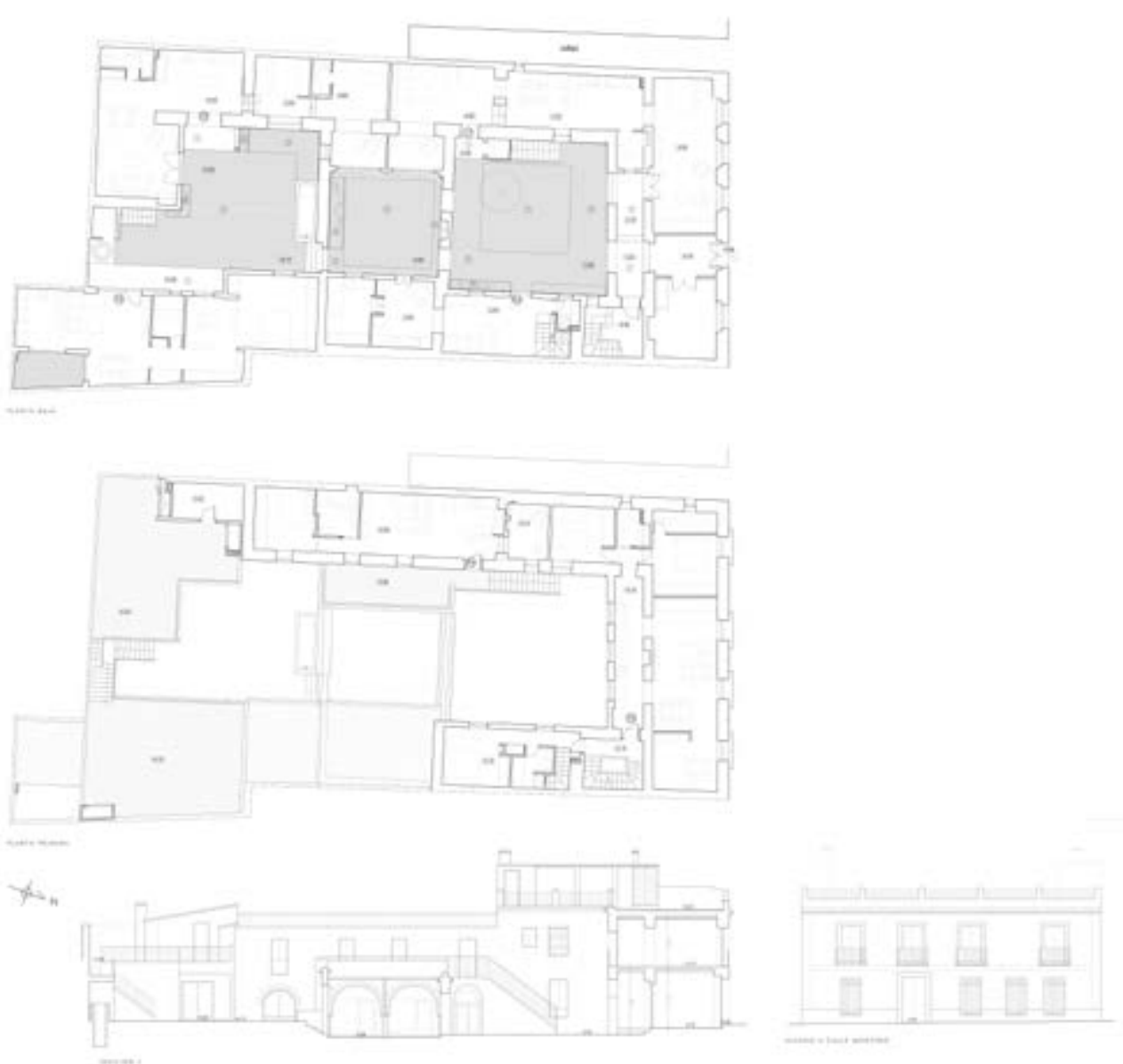

Imagen 10. Plantas, sección y alzado de la casa en calle Montero, primera cooperativa PAX - Fuente: Gaia Redaelli, Jacinta Ortiz, Carlos Anaya, arquitectos.

\section{Conclusiones}

La política de vivienda ha sido tradicionalmente, en España, una acción sectorial, ajena en muchos casos a un modelo urbano (que se evidencia en las viviendas vacías y en el consumo de suelo) y, posiblemente, ajena también a un modelo social (cuyos efectos son el número de desahucios) en la que administración y el sector han apostado por el crecimiento urbano. Parece el momento de un cambio de modelo productivo «de la especulación a la cultura de la rehabilitación» que permita también una gobernanza innovadora, multinivel y transversal, unas políticas urbanas y de vivienda basadas en la sostenibilidad ambiental y la cohesión social. Puesto que la crisis ha afectado 
de forma estructural a buena parte de la administración y, sobre todo, de la sociedad, es definitivamente oportuna no sólo una acción intersectorial de lo público, sino una alianza entre administración y economía social, entre ciudad y ciudadanía.

Si por parte de la administración pública es vital apostar por un parque público de vivienda en zonas centrales, que mantengan el mix social necesario, la economía social también debe entender su papel de activador de la ciudad consolidada, para reactivar áreas en riesgo de gentrificación.

De acuerdo a la afirmación según la cual «la ciudad se convierte, hoy, en el principal laboratorio en el que se buscan, se diseñan, se experimentan y se ponen a prueba soluciones locales a problemas globales» (Bauman, 2008, p. 29), PAX trabaja como gobernanza innovadora y experimental en este sentido y en diferentes escalas, al entender que se trata de una estrategia en curso en un laboratorio urbano a nivel local, que es Córdoba, pero que es transmisible a otras dimensiones y contextos similares para responder a la gentrificación como «estrategia urbana global». Si el solapamiento entre patrimonio material e inmaterial, en el caso del patio, parece especialmente apropiado, la estrategia busca seguir su implementación en la ciudad andaluza, pero también su transferencia a otros barrios o ciudades que, aun sin tener el patio como estructura urbana y social, se encuentran en condición similar y en incipiente gentrificación. Así, su adaptación a otros cascos históricos de ciudades mediterráneas es un reto inmediato de la estrategia, también a entornos no necesariamente céntricos, pero que necesitan densificarse por efecto de la despoblación, sobre todo donde la presencia de edificios de propiedad pública puede ayudar a la cesión de uso a entidades de la economía social, manteniendo la propiedad municipal y reduciendo la inversión por parte de los grupos ciudadanos.

A través de acciones de gobernanza innovadora en rehabilitación urbana e innovación social, la estrategia PAX busca actualizar el carácter urbano, ambiental, social y económico del patrimonio urbano como paso para entenderlo como un valor innegable de nuestro pasado y, a la vez, como una herramienta imprescindible para el futuro en una Europa inteligente, sostenible e inclusiva. Una Europa de las ciudades y de la ciudadanía. 


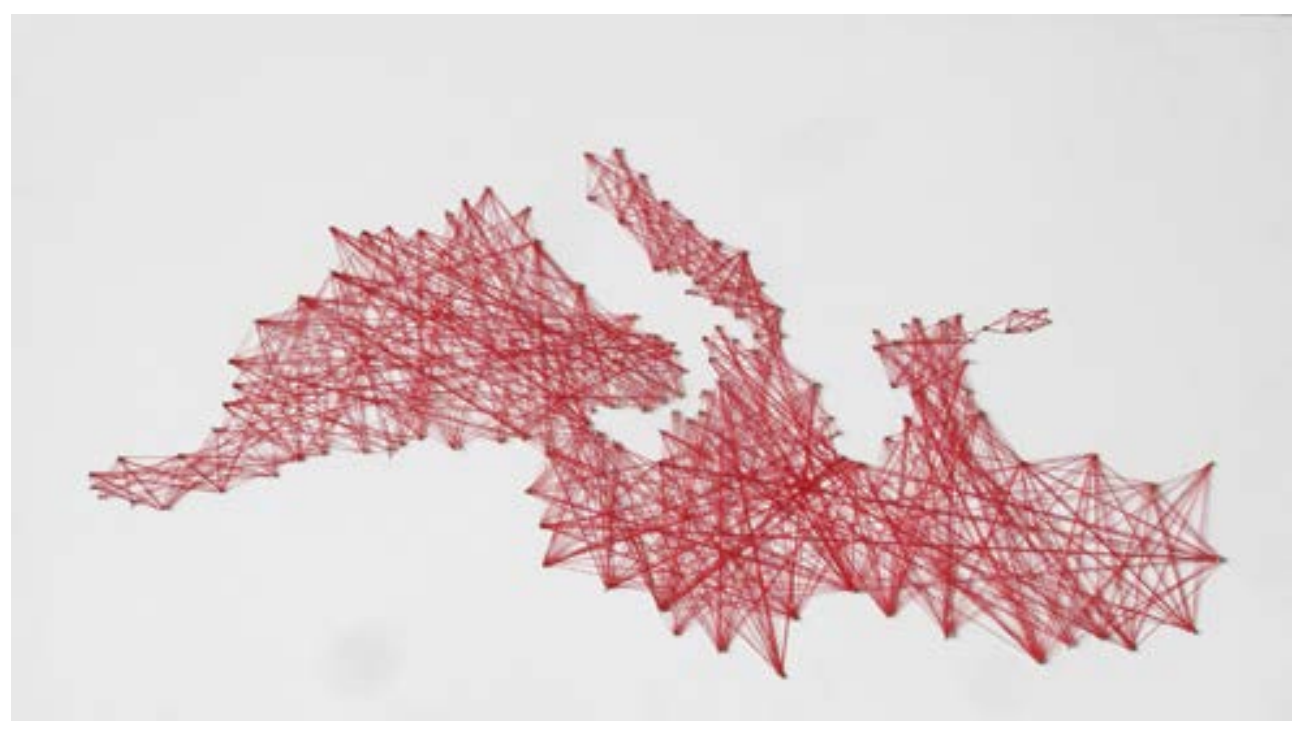

Imagen 11. Maqueta del Mediterráneo realizada con ocasión de la presentación de pax en la xv Biennal Internacional de Arquitectura de Venecia. Fuente: PAX.

\section{Referencias}

Anaya, C. (2020). Innovación social y Regeneración urbana. La experiencia PAX Patios de la Axerquía en la consolidación de una comunidad patrimonial, en Valor social, Fundación Finanzas Éticas, Barcelona, 2020 en https://valorsocial.info/innovacion-social-y-regeneracion-urbana-la-experiencia-pax-patios-de-la-axerquia-en-la-consolidacion-de-una-comunidad-patrimonial/

Annunziata, S. (2017). Anti-gentrificación nelle città (sud) europee. Quaderni n. 13, Urbanistica, mayo-agosto 2017.

Bauman, Z. (2008). Multiples culturas, una sola humanidad. Barcelona: СССв.

Battistella, A. (2017). La sostenibile leggerezza del limite. Techne - Journal of Technology for Architecture and Environment, 14. Firenze: University Press, 65-70

Franzoia, E. (2016). Cordova riscopre il patio mediterraneo. Abitare 558. 65-70

Consejo de Europa (2020). The Faro Convention: the way forward with heritage, Council of Europe, disponible en https://rm.coe.int/the-faroconvention-the-way-forward-with-heritage-brochure/16809e3627

HaRvey, D. (2012). La ciudad rebelde. Del derecho a la ciudad a la revolución urbana [The Rebellious City: From the Right to the City to the Urban Revolution]. Madrid: Akal. 
IndovinA, F. \& NeL·LO, O. (2017). Gentrification: disaster, necessity, opportunity? Notes for a critical use of the concept, en Albet, A. Benach, N.: Gentrification as a Global Strategy: Neil Smith and Beyond. London \& New York: Routledge.

Lees, L.; Slater, T. \& Wyly, E. (2010). The Gentrification Reader. New York: Routledge

Lees, L.; Bang Shin, H., López Morales, E. (2016). Planetary Gentrification. Cambridge: Polity

Lefebvre, H. (1975). El derecho a la ciudad. Barcelona: Penìnsula

Observatorio Metropolitano de Madrid (Ed) (2015). El mercado contra la ciudad. Globalización, gentrificación y politicas urbanas. Madrid: Traficante de sueños

Redaelli, G. (2018). Atlas de la A-Gentrificación. Políticas y medidas para la sostenibilidad social en las transformaciones urbanas en ciudades europeas. Papers 60. Barcelona: Institut de Estudi Metropolitans i Regionals de Barcelona, pp. 58-71.

- (2019). PAX - Patios de la Axerquía Urban Regeneration and Social Innovation in a Heritage Context. Built Heritage Volumen 3 n. 1/2019. Shanghai: Tongji University, pp. 91-104.

- (2020). Gobernanza cooperativa entre Ciudad y Ciudadanía, en Valor social, Fundación Finanzas Éticas, Barcelona 2020 en https://valorsocial.info/ gobernanza-cooperativa-entre-ciudad-y-ciudadania/

Rojas-Fernández J.; Galán-Marín C.; Roa-Fernández J. and Rivera-Gómez C. (2017). Correlations between GIS-Based Urban Building Densification Analysis and Climate Guidelines for Mediterranean Courtyards. Sustainability, 9. DOI: 10.3390/su9122255.

SECCHI, B. (2013). La città dei ricchi e la città dei poveri. Roma; Bari: Laterza.

Setris, S. (2014). Se Venezia muore. Torino: Einaudi.

Sмiтh, N. (2002) New Globalism, New Urbanism: Gentrification as Global Urban Strategy. Antipode 34 (3), pp. 434-457.

Synnes, K.; Artopoulos, G.; Smaniotto Costa, C.; Menezes, M. \& Redaelli, G. (2019). CyberParks Songs and Stories - Enriching Public Spaces with Localized Culture Heritage Material such as Digitized Songs and Stories. En Smaniotto Costa, C. \& Suklje-Erjavec, I., CyberParks - the interface between People, Places and Technology, (224-236). CyberParks Project. Springer: Heidelberg. 